\title{
Self-driven lattice-model Monte Carlo simulations of alloy thermodynamic properties and phase diagrams
}

\author{
$A$ van de Walle ${ }^{1}$ and $M$ Asta $^{1}$ \\ ${ }^{1}$ Materials Science and Engineering Department, Northwestern University, 2225 North Campus \\ Drive, Evanston, IL 60208, USA \\ E-mail: avdw@alum.mit.edu
}

Received 10 June 2002

Published 25 July 2002

Online at stacks.iop.org/MSMSE/10/521

\begin{abstract}
Monte Carlo (MC) simulations of lattice models are a widely used way to compute thermodynamic properties of substitutional alloys. A limitation to their more widespread use is the difficulty of driving a MC simulation in order to obtain the desired quantities. To address this problem, we have devised a variety of high-level algorithms that serve as an interface between the user and a traditional MC code. The user specifies the goals sought in a high-level form that our algorithms convert into elementary tasks to be performed by a standard MC code. For instance, our algorithms permit the determination of the free energy of an alloy phase over its entire region of stability within a specified accuracy, without requiring any user intervention during the calculations. Our algorithms also enable the direct determination of composition-temperature phase boundaries without requiring the calculation of the whole free energy surface of the alloy system.
\end{abstract}

\section{Introduction}

Monte Carlo (MC) simulations [1-4] of lattice models are a widely used method to compute thermodynamic properties of substitutional alloys. A lattice model MC simulation only requires, as an input, a so-called cluster expansion [5-7], which defines the energetics of the system by specifying the energy associated with any atomic arrangement on a given lattice. Cluster expansions are typically constructed from a fit to the energies of a set of structures calculated, for instance, from first-principles density-functional-theory calculations [8], thus enabling the prediction of thermodynamic properties even in the absence of experimental data.

In its simplest form, a cluster expansion specifies the energy difference between nearest-neighbour chemical bonds joining identical and distinct species in a binary alloy $\left(E_{\mathrm{AB}}-\left(E_{\mathrm{AA}}+E_{\mathrm{BB}}\right) / 2\right)$ and predicts compound formation energies from the number of each type of nearest-neighbour bond. However, this formalism can be extended [5] to allow for arbitrary interaction ranges and multibody interactions so that the energetics of the alloy can 
be modelled with an arbitrary accuracy by including a sufficient number of such interactions. It has been demonstrated that the energetic contributions arising from displacements of the atoms away from their ideal lattice sites $[6,7,9-14]$ can be accounted for within this framework. Moreover, by allowing temperature-dependent interactions, entropic contributions arising from vibrational and electronic excitations can be included as well (see [15] for a review and [16-19] for recent examples). Since long-range interactions are often needed to properly describe the energetics of an alloy system, MC simulations have become a preferred method to compute thermodynamics properties from cluster expansions, because it would be difficult to account for such long-range interactions within accurate mean-field methods, such as the cluster variation method (CVM) [20] while efficient algorithms to include even infinite-range interactions in MC simulations have been devised [21]. MC simulations are also conceptually simple to understand, straightforward to implement, and can be easily adapted to compute a wide variety of properties.

Given these attractive features, it is not surprising that MC simulations of lattice models with cluster expansions determined from first-principles calculations have been employed in a variety of contexts for metallic, semi-conductor and ceramic systems, including the computation of: composition-temperature phase diagrams, thermodynamic properties of stable and metastable phases, short-range order in solid solutions, thermodynamic properties of planar defects (including surfaces or antiphase and interphase boundaries) and the morphology of precipitate microstructures [6,7,11,22-25].

With the steadily increasing availability of computational resources, MC simulations of lattice models are likely to become even more prevalent in computational material science modelling. Despite the technological advances in computational hardware, the human time needed to select the appropriate simulation parameters and to analyse the data has remained essentially constant over the years. We are now in a situation where the main limitation to the inclusion of atomistic MC simulations in standard thermodynamic software toolkits is not the lack of computational resources, but rather the difficulty of controlling a MC simulation in order to obtain the desired quantities.

To address this problem, we have devised a variety of high-level algorithms that serve as an interface between the user and a traditional MC code. The user specifies the goals sought in a high-level form that our algorithm converts into elementary tasks to be performed by a standard MC code. For instance, our algorithms permit the determination of the free energy of a phase over all of its region of stability within a specified accuracy, without requiring any user intervention during the calculations. The proposed algorithms have been implemented in an easy-to-use software package [26].

In an effort to make this paper self-contained, we first recall the basic theoretical results underlying semi-grand-canonical MC simulations, which are especially suited for determination of thermodynamic properties of alloys. We then proceed to describe the algorithms that enable the automated calculation of thermodynamic quantities from MC simulations. These algorithms address the following three questions. (i) How to determine when a MC simulation has run for a sufficiently long time to provide the desired quantities within a given target precision? (ii) How to detect phase transitions? (iii) How to efficiently determine the composition-temperature phase boundaries of a phase diagram? We then provide a series of examples of applications that illustrate the features of our algorithms.

\section{Semi-grand-canonical MC}

A very convenient way to obtain thermodynamic information regarding an alloy system is to perform MC simulations which sample a semi-grand-canonical ensemble, also known as 
the transmutation ensemble. In this ensemble, the energy and concentration of an alloy with a fixed total number of atoms $(N)$ are allowed to fluctuate while temperature and chemical potentials are externally imposed. Although the results of this paper can be extended to general multicomponent systems, we are considering the case of binary alloys, for the benefit of notational simplicity. In an A-B alloy, without loss of generality, only the composition $x$ of element $\mathrm{A}$ and the difference in chemical potential between the two species $\mu=\mu_{\mathrm{A}}-\mu_{\mathrm{B}}$ needs to be specified. For conciseness, we simply refer to the quantity $\mu$ as the "chemical potential' and $x$ as the concentration.

The natural thermodynamic potential $\phi$ (expressed per atom) associated with the semigrand-canonical ensemble can be defined in terms of the partition function of the system as follows:

$$
\phi(\beta, \mu)=-\frac{1}{\beta N} \ln \left(\sum_{i} \exp \left(-\beta N\left(E_{i}-\mu x_{i}\right)\right)\right),
$$

where $E_{i}$ and $x_{i}$ are, respectively, the internal energy (per atom) and the concentration of state $i$, while $\beta=1 /\left(k_{\mathrm{B}} T\right)$ is the reciprocal of the temperature $T$ and $k_{\mathrm{B}}$ is Boltzmann's constant. The thermodynamic potential $\phi$ is related to the Helmholtz free energy $F$ through $\phi=F-\mu x$.

The potential $\phi$ can also be defined by the following total differential:

$$
\mathrm{d}(\beta \phi)=(E-\mu x) \mathrm{d} \beta-\beta x \mathrm{~d} \mu,
$$

where $E$ and $x$ are the system's average internal energy (per atom) and concentration. Equation (2) enables the calculation of the thermodynamic function $\phi(\beta, \mu)$ through thermodynamic integration:

$$
\beta_{1} \phi\left(\beta_{1}, \mu_{1}\right)=\beta_{0} \phi\left(\beta_{0}, \mu_{0}\right)+\int_{\left(\beta_{0}, \mu_{0}\right)}^{\left(\beta_{1}, \mu_{1}\right)}(E-\mu x,-\beta x) \mathrm{d}(\beta, \mu),
$$

where the integral is performed along a continuous path joining points $\left(\beta_{0}, \mu_{0}\right)$ and $\left(\beta_{1}, \mu_{1}\right)$ that does not encounter a phase transition. Semi-grand-canonical MC simulations can be directly used to determine the values of $E$ and $x$ for any given $\beta$ and $\mu$.

The potential at the initial point $\phi\left(\beta_{0}, \mu_{0}\right)$ is usually chosen to be a convenient point where $\phi$ can be computed analytically. One possible starting point is the high-temperature limit [11],

$$
\beta \phi(\beta, \mu) \rightarrow-\ln (2) \quad \text { as } \beta \rightarrow 0,
$$

where the limit is taken while keeping $\mu$ finite (implying that concentration $x$ converges to $\frac{1}{2}$ as $\beta \rightarrow 0$ ). Another possible starting point is in the limit of low temperature at a chemical potential stabilizing a given ground state $g$. In this limit, a 'single-spin flip' low-temperature expansion (LTE) $[11,27,28]$ can be used to obtain

$$
\phi(\beta, \mu)=E_{\mathrm{g}}-\mu x_{\mathrm{g}}-\frac{1}{\beta N} \sum_{s} \exp \left(-\beta\left(\Delta \varepsilon_{s, \mathrm{~g}}-\mu \Delta \eta_{s, \mathrm{~g}}\right)\right),
$$

where $E_{\mathrm{g}}$ is the energy (per atom) of ground state $\mathrm{g}$ with composition $x_{g}$, while $\Delta \varepsilon_{s, \mathrm{~g}}$ is the variation in the system's total energy $(N E)$ associated with changing the identity of the atom sitting at site $s$ in ground state $\mathrm{g}$, and $\Delta \eta_{s, \mathrm{~g}}$ is the variation in $(N x)$ associated with the same change. This infinite sum can be reduced to a finite number of terms by making use of the translational periodicity of the ground state.

In order to avoid lengthy MC simulations, it is useful to possess a criterion to find the highest possible temperature where the LTE is accurate. One attractive possibility is to compute $\phi$ both from the LTE and from a mean field approximation (MFA). As long as both approaches give the same result within a user specified tolerance, we can be confident that 'multiple-spin flip events' are rare and that the LTE has the desired level of precision. 


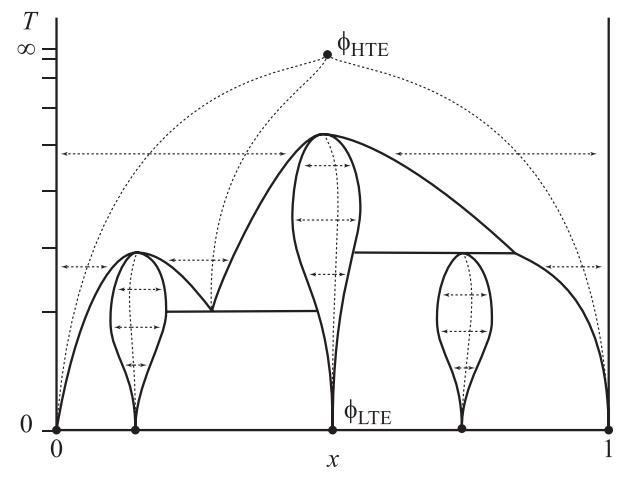

Figure 1. Example of paths of integration to determine the potential $\phi(\beta, \mu)$ of every phase. The starting points are given by either the LTE or the high-temperature expansion (HTE).

Using the LTE for each ground state of the system as a starting point to evaluate equation (3), one can map out the whole potential surface $\phi^{\alpha}(\beta, \mu)$ associated with any given ordered phase $\alpha$. Similarly, the high-temperature limit can be used as a starting point to obtain $\phi^{\alpha}(\beta, \mu)$ for the disordered phase. This process is illustrated in figure 1 . Once $\phi^{\alpha}(\beta, \mu)$ has been determined for all phases, the boundary between two given phases $\alpha$ and $\gamma$ can be located by identifying the locus $\Lambda^{\alpha \gamma}=\left\{(\beta, \mu): \phi^{\alpha}(\beta, \mu)=\phi^{\gamma}(\beta, \mu)\right\}$ where the potential surfaces intersect. At such intersections $(\beta, \mu) \in \Lambda^{\alpha \gamma}$, the concentrations $x^{\alpha}$ and $x^{\gamma}$ of the two phases in equilibrium are simply given by the slopes of the potential surface at the point of intersection:

$$
\begin{aligned}
& x^{\alpha}=-\frac{\partial \phi^{\alpha}(\beta, \mu)}{\partial \mu}, \\
& x^{\gamma}=-\frac{\partial \phi^{\gamma}(\beta, \mu)}{\partial \mu} .
\end{aligned}
$$

The calculations of thermodynamic quantities based on semi-grand-canonical MC simulations offer several advantages over their canonical counterparts.

(a) For a given value of the control variables $(\beta, \mu)$, the thermodynamic equilibrium of the system is never a phase-separated mixture, implying that the calculated quantities always reflect the properties of pure phases, free of interfacial contributions that would otherwise not be negligible due to the finite size of the simulation cell. (Note that the MC simulation cell must be commensurate with the periodicity of the equilibrium structure for this property to hold.)

(b) The potential $\phi$ can be very directly determined through a simple thermodynamic integration procedure (equation (3)) where each required quantity takes the form of a thermodynamic average of computationally inexpensive quantities ( $E$, which must already be computed in order to perform the simulation, and $x$, which is trivial to obtain).

(c) Phase boundaries can be located by looking for intersections between curves, a criterion which is somewhat simpler to implement than the common tangent construction (although both methods are formally equivalent).

\section{Algorithms}

While the methods described in the previous section, in principle, enable the determination of thermodynamic quantities from MC simulations, a number of practical issues need to be addressed before this process can be fully automated. 
A typical MC code requires a substantial amount of user intervention and relies on the user's physical intuition to identify when a simulation is successful and reliable. The type of user interventions needed include:

(a) providing a variety of input parameters controlling the computational accuracy that need to be determined by trial and error;

(b) requiring the user to monitor the simulation periodically to ensure that the system has not undergone an unexpected phase transformation;

(c) performing a substantial amount of post-processing in order to obtain a phase diagram.

In this section we introduce algorithms to perform these tasks automatically and provide a formal justification of their applicability.

\subsection{Reaching a target precision}

Three parameters control the precision of a thermodynamic quantity computed through MC simulations: the simulation cell size, the equilibration time and the averaging time. While the analysis of the effect of simulation cell size on the precision is beyond the scope of this paper, this section provides a sound basis for the selection of the two remaining parameters. The equilibration time is the time the system takes to reach thermodynamic equilibrium from a given initial configuration. Once equilibrium is reached, the instantaneous value of a given quantity (e.g. the energy) then needs to be averaged over a certain number of MC steps in order for its average to be sufficiently accurate. This defines the averaging time, which is the first parameter we will focus on.

3.1.1. Averaging time. While the approach presented in this section relies on standard statistical results, it is nevertheless included for the purpose of introducing our notation and clarifying the assumptions made. Consider a microscopic quantity taking the value $Q_{t}$ at step $t$ and whose expectation value is equal to the desired thermodynamic quantity $Q$. If $L$ observations of $Q_{t}$ are available, a natural estimator of $Q$ is the average:

$$
\bar{Q}_{[1, L]}=\frac{1}{L} \sum_{t=1}^{L} Q_{t} .
$$

The precision of this statistical quantity can be quantified by calculating its variance. In general, the variance of $\bar{Q}_{[1, L]}$ is given by

$$
\operatorname{Var}\left(\bar{Q}_{[1, L]}\right)=\frac{1}{L^{2}} \sum_{t=1}^{L} \sum_{s=1}^{L} V_{|t-s|}
$$

where $V_{|t-s|}$ is the covariance between $Q_{t}$ and $Q_{s}$. The fact that the sequence $Q_{t}$ is stationary when the system has reached thermodynamic equilibrium enables a simple estimator $\hat{V}_{l}$ of the covariance structure $V_{l}$ of the sequence through the equation

$$
\hat{V}_{l}=\frac{1}{L-l} \sum_{t=1}^{L-l} Q_{t} Q_{t+l}-\bar{Q}_{[1, L]}^{2} .
$$

While equation (9) is very general, it is also very computationally intensive, requiring on the order of $L^{2}$ operations to estimate the variance of $\bar{Q}_{[1, L]}$. Following most of the literature on MC simulation, we consider the more computationally tractable special case obtained when the covariance structure $V_{l}$ is assumed to be of the form

$$
V_{l}=V \rho^{-|l|} \text {. }
$$


This approximation can be motivated as follows. A Markov chain can be represented by a linear superposition of components with exponentially decaying autocorrelation function. Each component is characterized by a certain correlation length and the component with the longest correlation length will typically give, by far, the largest contribution to the variance. Considering only the most slowly decaying component should thus provide a good approximation to the true variance of the quantity of interest.

Under the assumption that the averaging time is much larger than the longest correlation time ${ }^{2}$, 'boundary' effects can be neglected and equation (9) then reduces to

$$
\operatorname{Var}\left(\bar{Q}_{[1, L]}\right)=\frac{1}{L^{2}} \sum_{t=1}^{L} \sum_{l=-\infty}^{\infty} V \rho^{|l|} .
$$

Evaluating the geometric sum then yields

$$
\operatorname{Var}\left(\bar{Q}_{[1, L]}\right)=\frac{V}{L}\left(\frac{1+\rho}{1-\rho}\right) .
$$

Note that, since $\rho=\exp (-1 / \tau)$ where $\tau$ is the characteristic relaxation time, equation (13) can be written, in the limit of large $\tau$, in the more familiar form $\operatorname{Var}\left(\bar{Q}_{[1, L]}\right)=2 \tau V / L$.

While $V$ can be directly estimated from equation (10), setting $l=0$, the parameter $\rho$ can be found in a variety of ways. We found the following algorithm to be both fast and accurate: find the time interval $l^{*}$ such that $\hat{V}_{l^{*}} / \hat{V}_{0}=\frac{1}{2}$ and then set $\rho=2^{-1 / l^{*}}$. This approach offers the important advantage that it uses, as an input, only the correlation between relatively distant values of $Q_{t}$. This ensures that $\rho$ will be determined by the most slowly decaying components of the Markov process, which are the ones that the functional form $V \rho^{|l|}$ was meant to model. It is also a very fast algorithm, since a simple bracketing algorithm allows the determination of $\rho$ in about $L \log L$ operations. In the event that there are more than one $l^{*}$ satisfying the equality, we chose the smallest one. The rationale behind this choice is that there is a small but nonzero chance that the estimated covariances are such that $\hat{V}_{l} / \hat{V}_{0}=\frac{1}{2}$ although the true covariances are not such that $V_{l} / V=\frac{1}{2}$. Choosing the smallest $l^{*}$ makes the procedure less sensitive to these spurious events since the probability of occurrence of one such event increases with the range of values of $l$ considered.

In short, the averaging time $L$ needed to reach a given precision can be found by computing the variance of $\bar{Q}_{[1, L]}$ through equation (13) periodically and stopping the simulation when the variance goes below a user specified threshold:

$$
\operatorname{Var}\left(\bar{Q}_{[1, L]}\right) \leqslant\left(\frac{p}{z_{\alpha}}\right)^{2},
$$

where $p$ is the target precision on the value of $Q$ while

$$
z_{\alpha}=\sqrt{2} \operatorname{erf}^{-1}(1-\alpha)
$$

is the critical value associated with the desired level of confidence $\alpha$. For instance, if the error on $Q$ is required to be less than $10^{-3}$ with a probability of $99 \%$, then $p=10^{-3}, \alpha=0.01$ and $z_{\alpha}=2.576$.

3.1.2. Equilibration time. When devising a criterion to determine whether a simulation has reached thermodynamic equilibrium, approaches based on identifying correlation lengths can be unreliable because the system may be very far from equilibrium during equilibration. For

2 This assumption should not be a concern because (i) it tends to be violated only for averaging times such that the desired target precision on $\bar{Q}$ has not yet been reached, and (ii) when it is violated, our expression overestimates the true variance. 
(a) $\uparrow Q$

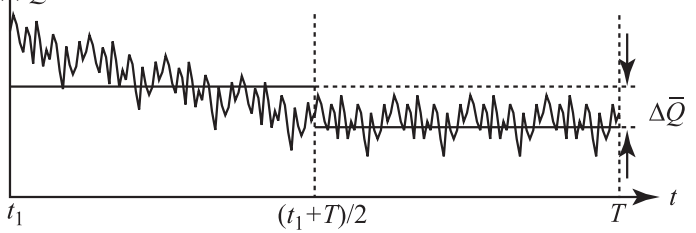

(b) $\uparrow Q$

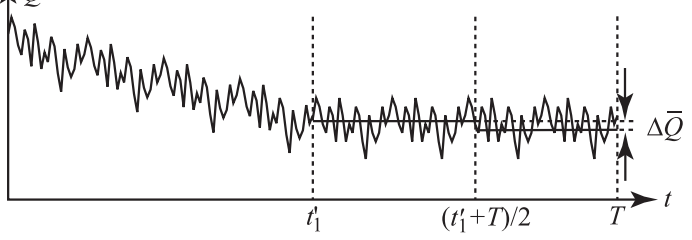

Figure 2. Criterion for testing whether thermodynamic equilibrium has been reached in a MC simulation. In $(a)$, the two blocks of data have significantly different means, implying that the hypothesis that equilibrium has been reached at $t_{1}$ is rejected. In $(b)$, the two blocks of data do have similar means, indicating that the hypothesis that equilibrium has been reached at $t_{1}^{\prime}$ cannot be rejected.

this reason, we employ a relatively simple, and yet very robust approach that is based on the main defining characteristic of thermodynamic equilibrium. If the average of the quantity $Q_{t}$ between steps $t_{1}$ and $t_{2}$ is not statistically significantly different from the average of $Q_{t}$ between steps $t_{2}$ and $t_{3}$, then the hypothesis that equilibrium has been reached at step $t_{1}$ and beyond cannot be rejected. Of course, the power of this test increases as $t_{2}-t_{1}$ and $t_{3}-t_{2}$ increase and this definition of equilibrium thus depends on the target precision $p$ we seek to reach. This is to be expected since, strictly speaking, thermodynamic equilibrium is never reached in a simulation of a finite length. Any definition of equilibrium must include an arbitrary cutoff and it is natural to choose this cutoff to be a function of the precision we require.

Both the equilibration and averaging time needed to reach a precision $p$ can thus be determined through an algorithm that can be outlined as follows (refer to figure 2). Let $L$ denote the number of samples of $Q_{t}$ collected so far. Consider a range of values of $t_{1} \in[1, L]$ and for each, check whether

$$
\Delta \bar{Q} \equiv\left|\bar{Q}_{\left[t_{1},\left(t_{1}+L\right) / 2\right]}-\bar{Q}_{\left[\left(t_{1}+L\right) / 2, L\right]}\right| \leqslant p,
$$

where $Q_{\left[t_{1}, t_{2}\right]}$ denotes the average of $Q_{t}$ for $t \in\left[t_{1}, t_{2}\right]$. Then, verify that $\operatorname{Var}\left(\bar{Q}_{\left[t_{1}, L\right]}\right)$ satisfies equation (14). If both conditions are satisfied, then $\bar{Q}_{\left[t_{1}, L\right]}$ provides an estimate of $Q$ with a precision $p$ and with a confidence level $\alpha$.

In its simplest form, the implementation of this algorithm involves storing all samples of $Q_{t}$ collected so far and requires a loop over values of $t_{1}$ which make the complexity of the algorithm $\mathrm{O}\left(L^{2}\right)$. Various techniques help reduce the resources required. First, sampling $Q_{t}$ every $\mathrm{MC}$ pass $^{3}$, instead of at every spin flip, results in essentially no loss in precision since the correlation length of the simulation typically extends much beyond a MC pass. Second, one can limit the search for $t_{1}$ to values that are integer multiple of some block size $L_{\mathrm{b}}$ that is allowed to increase linearly with the total number of MC passes $L$. In this fashion, the number of operations needed to determine $t_{1}$ does not increase as the simulation progresses. The value of $t_{1}$ thus found by a search constrained to block boundaries differs at most by $L_{\mathrm{b}}$ from the $t_{1}$ which would have been found without block constraints. In the worst case, the simulation may

3 A MC pass consists of a number of attempted spin flips equal to the number of lattice sites in the simulation cell. 


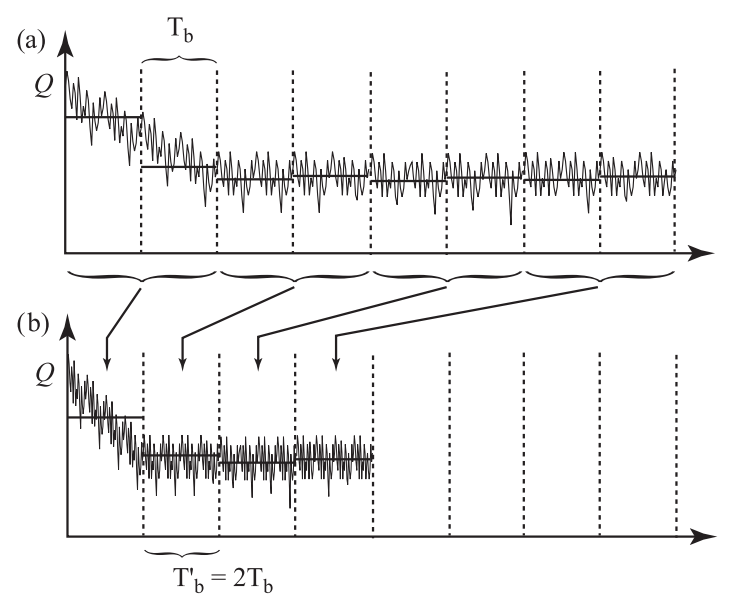

Figure 3. Blockwise storage scheme. (a) Only the mean variance and correlations within blocks of width $L_{\mathrm{b}}$ needs to be stored (the mean is represented by a thick horizontal line). (b) As the simulation runs, blocks are periodically merged so that storage requirements do not grow.

have to be run for an additional $L_{\mathrm{b}}$ passes and it follows that the length of the simulation will be at most $\left(1+L_{\mathrm{b}} / L\right)$ larger than the optimal computational time that would have been needed without block constraints. A computationally convenient way to implement this scheme is to multiply the block size $L_{\mathrm{b}}$ by two whenever the total number of passes $L$ doubles. Thanks to this approach, the entire history of values of $Q_{t}$ does not need to be stored, only block averages of $\bar{Q}, V$ and $\rho$ are needed, as illustrated in figure 3. When the block size doubles, the values of $\bar{Q}, V$ and $\rho$ for two consecutive blocks can be combined to obtain the corresponding value for the new block having twice the length ${ }^{4}$.

\subsection{Detecting phase transitions}

Phase transitions can be identified by locating singularities in some function $Q(C)$, where $Q$ is some thermodynamic quantity (such as energy, concentration or an order parameter) and $C$ is some control variable (such as temperature or chemical potential). In this section, we propose a simple algorithm to perform the detection of such singularities while accounting for the fact that quantities obtained from MC are intrinsically noisy. Our algorithm is applicable to the detection of both first-order and second-order transitions, although the power of the test is clearly higher for first-order transitions.

An analytic function has the property that the knowledge of its shape in some finite interval enables the prediction of its value outside of that interval. The failure of this extrapolation procedure in the presence of a singularity permits its detection. Formally, an analytic function can be extrapolated using a Taylor series. We approximate this operation by fitting the output of the MC simulation by a polynomial.

Consider $n+1$ estimates $\bar{Q}_{1}, \ldots, \bar{Q}_{n+1}$ of the quantity $Q(C)$ obtained for a sequence of values of the control variable $C_{1}, \ldots, C_{n+1}$. The general principle behind our singularity detection procedure is to fit a polynomial through the first $n$ points and if the observed value

4 The only drawback is that the resulting estimate of $\rho$ does not make use of all the available data points, because the product $Q_{t} Q_{s}$ for $t$ and $s$ belonging to different blocks cannot be determined from within-block averages. Although it is inefficient, our estimate of $\rho$ is nevertheless unbiased and the loss of efficiency is negligible if the block size is large relative to the correlation time. 
$\bar{Q}_{n+1}$ is far from the value predicted from the polynomial fitted to the previous data, a singularity must be present between point $n$ and $n+1$ (see figure 4). To make this algorithm practical, two questions need to be answered. First, how do we choose the order of the polynomial to be fitted? Second, how do we select the maximum allowable prediction error that can be tolerated without claiming the presence of a singularity?

To answer the first question, we make use of the well-known cross-validation criterion $[29,30]$ : choose the order $k$ of the polynomial that minimizes the quantity

$$
C V=\frac{1}{n} \sum_{i=1}^{n}\left(\bar{Q}_{i}-Q_{i}^{*}\right)^{2},
$$

where $Q_{i}^{*}$ is the value of $\bar{Q}_{i}$ predicted from a polynomial least-squares fit to the remaining $n-1$ points: $\bar{Q}_{1}, \ldots, \bar{Q}_{i-1}, \bar{Q}_{i+1}, \ldots, \bar{Q}_{n}$. Intuitively, this criterion tests the predictive power of the polynomial using the points $\bar{Q}_{1}, \ldots, \bar{Q}_{n}$ before we attempt to use it for the prediction of $\bar{Q}_{n+1}$. Once the order $k$ of the polynomial has been chosen, the standard theory of least squares [31] provides the distribution of the predicted value $Q_{n+1}^{*}$, which can be used to determine whether the prediction error is statistically significantly different from zero. For $i=1, \ldots, n$ and $j=1, \ldots, k+1$, let

$$
\begin{aligned}
& X_{i j}=\left(C_{i}\right)^{j-1}, \\
& Y_{i}=\bar{Q}_{i},
\end{aligned}
$$

so that the equation defining the least-squares problem can be written in matrix form as $Y=X a+\varepsilon$, where $\varepsilon$ is an $n \times 1$ vector of error terms. The vector $a$ of the coefficients of the polynomial can be estimated by

$$
\hat{a}=\left(X^{\mathrm{T}} X\right)^{-1} X^{\mathrm{T}} Y
$$

while the covariance matrix of $\hat{a}$ is given by

$$
V=\sigma^{2}\left(X^{\mathrm{T}} X\right)^{-1}
$$

where $\sigma^{2}$ is the variance of the residuals $\varepsilon_{i}$ of the fit. The quantity $\sigma^{2}$ can be estimated in two asymptotically equivalent ways. Equation (13) provides an estimate of the variance of any of the $\bar{Q}_{i}$, which can be averaged over $i$ to yield a single, more accurate estimate:

$$
\hat{\sigma}^{2}=\frac{1}{n} \sum_{i=1}^{n} \operatorname{Var}\left(\bar{Q}_{i}\right) .
$$

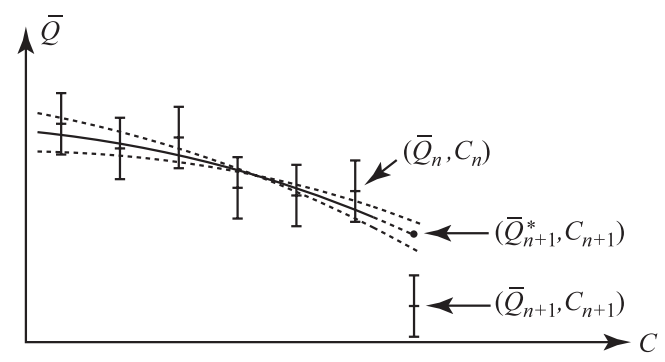

Figure 4. Criterion for detecting a phase transition. If the sequence $\left(\bar{Q}_{n}, C_{n}\right)$ predicts a value of $\left(\bar{Q}_{n+1}^{*}, C_{n+1}\right)$ but the actual value $\left(\bar{Q}_{n+1}, C_{n+1}\right)$ is statistically significantly different, then a phase transition must have occurred. Both the noise in the prediction (represented by dashed lines) and the noise in $\bar{Q}_{n+1}$ (represented by an error bar) must be taken into account to determine statistical significance. 
Alternatively, the residuals of the least-squares fit can be used:

$$
\hat{\sigma}^{2}=\frac{\|Y-X a\|^{2}}{n-k-1} .
$$

While equation (22) is more accurate (especially if $n$ is small), because it makes use of additional information, equation (23) is simpler to implement as it minimizes the amount of information that needs to be transferred between the MC simulation code and the phase transition detection routine.

The predicted value of $Q$ for point $n+1$ is then given by

$$
Q_{n+1}^{*}=x^{\mathrm{T}} \hat{a},
$$

where $x_{j}=\left(C_{n+1}\right)^{j-1}$ for $j=1, \ldots, k+1$, while the variance of this prediction is given by

$$
v=x^{\mathrm{T}} V x \text {. }
$$

If there is no singularity, the variance of the difference between $\bar{Q}_{n+1}$ and $Q_{n+1}^{*}$ is $v+\sigma^{2}$ because (i) the variance of $Q_{n+1}^{*}$ is $v$ while the variance of $\bar{Q}_{n+1}$ is $\sigma^{2}$, (ii) $\bar{Q}_{n+1}$ and $Q_{n+1}^{*}$ are independent. An asymptotically valid statistical test thus consists in rejecting the hypothesis that there is no phase transition if the prediction error $\left|\bar{Q}_{n+1}-Q_{n+1}^{*}\right|$ is such that ${ }^{5}$

$$
\left|\bar{Q}_{n+1}-Q_{n+1}^{*}\right| \geqslant z_{\alpha} \sqrt{v+\sigma^{2}}
$$

For $z_{\alpha}$ defined as in equation (15), the probability that a phase transition is incorrectly identified is less than $\alpha$. The relevant quantities are represented in figure 4.

The equations presented above rely on the assumption that the deviations away from the true thermodynamic quantity, $\left(\bar{Q}_{i}-Q\left(C_{i}\right)\right)$ for $i=1, \ldots, n+1$, are statistically independent. This is an appropriate assumption if the system is given sufficient time to equilibrate every time the value of the control variable $C$ changes. Our method also assumes that the deviations $\left(\bar{Q}_{i}-Q\left(C_{i}\right)\right)$ all have the same variance. Both of these requirements are automatically satisfied if the points $\bar{Q}_{1}, \ldots, \bar{Q}_{n+1}$ are generated using the algorithm presented in section 3.1.

For the sole purpose of locating phase transitions, one particular type of thermodynamic function enables an especially sensitive test for phase transitions: order parameters. Unfortunately, order parameters that are invariant under any possible symmetry operation of the lattice are often expensive to compute. However, if one always traverses phase transitions from an ordered phase toward a disordered phase (or another ordered phase), the task is dramatically simplified. When a MC cell is initialized with one specific ground state (i.e. an ordered phase) and before a phase transition is reached, there is a vanishing probability that the system will fluctuate enough to reach a state that is different but symmetrically equivalent to the original state. Thus, an order parameter can be computed by simply keeping track of the number of sites that host an atomic specie that is different from the specie found at the same site at initialization. There is no need to check for every possible symmetry-related ordered configuration.

The algorithms just presented are especially useful when one desires to construct the potential surface $\phi(\beta, \mu)$ of a phase (using thermodynamic integration over the paths depicted in figure 1) without requiring the user to manually determine when to stop the thermodynamic integration along a given direction because a phase transition has occurred. The automated

5 Note that $Q_{n+1}^{*}$ must be an unbiased predictor of $Q\left(C_{n+1}\right)$ for this test to be valid, which is not the case for finite $n$ due to the finite order of the approximating polynomial. Fortunately, the bias (squared) goes to zero as $n \rightarrow \infty$ and eventually becomes negligible relative to $\sigma^{2}$, ensuring the asymptotic validity of the procedure. Note that the variance $v$ of the prediction is also negligible asymptotically relative to $\sigma^{2}$. We nevertheless include the variance term $v$ in equation (26) in order to improve the accuracy of the test for finite $n$, since we do have an estimator of the variance (unlike the bias). 
construction of such free energy surfaces provides a very natural pathway through which an atomistic MC simulation can be used to provide input to CALPHAD-type calculations based on phenomenological free energy models.

\subsection{Phase boundary tracing}

When one is solely interested in determining a system's phase diagram, it would be computationally advantageous to be able to follow the boundary of a phase without first calculating the potential $\phi$ over the whole region where this phase is stable. In this section, we describe how, in the case of a first-order transition, the entire boundary of a two-phase equilibrium can be determined, starting from a single point where a transition is known to occur. We first focus on a simple method that neglects the unavoidable presence of statistical noise in MC simulations before describing how the presence of this noise can be handled.

Consider a value of the reciprocal temperature $\beta$ and of the chemical potential $\mu$ that is known to stabilize a phase-separated mixture between phases $\alpha$ and $\gamma$. The knowledge of such a pair $(\beta, \mu)$ could be provided, for instance, by the phase transition detection algorithm presented in the previous section, assuming that the hysteresis loop of the phase transition is sufficiently narrow (which tends to be case at high temperature). Alternatively, if $T=0$, the chemical potential that simultaneously stabilizes two ground states $\alpha$ and $\gamma$ can be found by a simple common tangent construction from the knowledge of the energy and composition of each ground state. At such a two-phase equilibrium point, the potentials $\phi$ of each phase are equal. Our task is to find, as reciprocal temperature $\beta$ changes by an amount $\mathrm{d} \beta$, the changes in chemical potential needed to preserve the equality between each phase's thermodynamic potential. By iterating this process, one can define a whole path $\mu(\beta)$ that traces out the value of the chemical potential at the phase transition as temperature changes, and thus find the concentrations $x_{\alpha}$ and $x_{\gamma}$ of each phase in equilibrium as a function of temperature. The differential equation whose solution provides the desired path $\mu(\beta)$ can be found by taking the total differential of the equation $\beta \phi^{\alpha}=\beta \phi^{\gamma}$ following from the identity between the potentials $\phi$ of each phase:

$$
\begin{aligned}
\left.\frac{\partial\left(\beta \phi^{\alpha}\right)}{\partial \beta}\right|_{\mu} \mathrm{d} \beta & +\left.\frac{\partial\left(\beta \phi^{\alpha}\right)}{\partial \mu}\right|_{\beta} \mathrm{d} \mu=\left.\frac{\partial\left(\beta \phi^{\gamma}\right)}{\partial \beta}\right|_{\mu} \mathrm{d} \beta+\left.\frac{\partial\left(\beta \phi^{\gamma}\right)}{\partial \mu}\right|_{\beta} \mathrm{d} \mu \\
& \Rightarrow\left(E^{\alpha}-\mu x^{\alpha}\right) \mathrm{d} \beta-\beta x^{\alpha} \mathrm{d} \mu=\left(E^{\gamma}-\mu x^{\gamma}\right) \mathrm{d} \beta-\beta x^{\gamma} \mathrm{d} \mu \\
& \Rightarrow \frac{\mathrm{d} \mu}{\mathrm{d} \beta}=\frac{\left(E^{\gamma}-E^{\alpha}\right)}{\beta\left(x^{\gamma}-x^{\alpha}\right)}-\frac{\mu}{\beta},
\end{aligned}
$$

where $E^{\alpha}$ and $x^{\alpha}$ are the energy and concentration of phase $\alpha$ at reciprocal temperature $\beta$ and chemical potential $\mu$ (and similarly for phase $\gamma$ ). All the quantities needed can be directly obtained from MC simulations. Thanks to the fact that both phases are metastable for values of the chemical potential in the neighbourhood of the true phase transition, it is possible to obtain $E$ and $x$ for both phases at the same chemical potential. A very efficient way to implement this algorithm is to keep two simulations in memory at the same time-one for the phase $\alpha$ and one for the phase $\gamma$. In this fashion, the final configuration of the simulation of phase $\alpha$ at reciprocal temperature $\beta$ can be used as an initial configuration for the simulation of phase $\alpha$ at temperature $\beta+\mathrm{d} \beta$ (and similarly for phase $\gamma$ ). The long equilibration time associated with the crossing of a phase transition is thus avoided. Interestingly, this algorithm can be directly used even when the two phases in equilibrium are based on a completely different parent lattice.

We now address the issue that the quantities obtained from MC are contaminated by noise. The presence of noise causes the solution to equation (29) calculated from MC to deviate 
from the true path $\mu(\beta)$. This eventuality brings up two questions. First, is the solution to equation (29) stable or unstable with respect to the presence of small perturbations? Second, what can one do if the noise becomes so large that the calculated chemical potential leaves the region where both phases are metastable?

To answer the first question, consider a perturbation $\varepsilon(\beta)$ of the chemical potential away from its value $\mu(\beta)$ at the phase transition at reciprocal temperature. We now calculate how this error is further propagated when solving equation (29). To the first order, the difference between the potential $\phi$ of the two phases is $\left(x^{\gamma}(\beta)-x^{\alpha}(\beta)\right) \varepsilon(\beta)$. As temperature changes, equation (29) updates $\mu$ so as to preserve this difference. We thus have the equality

$$
\left(x^{\gamma}(\beta)-x^{\alpha}(\beta)\right) \varepsilon(\beta)=\left(x^{\gamma}(\beta+\mathrm{d} \beta)-x^{\alpha}(\beta+\mathrm{d} \beta)\right) \varepsilon(\beta+\mathrm{d} \beta) .
$$

Taking the limit of infinitesimal $\mathrm{d} \beta$ and rearranging yields

$$
\frac{\mathrm{d} \varepsilon}{\mathrm{d} \beta}=-\varepsilon \frac{\mathrm{d}}{\mathrm{d} \beta} \ln \left|x^{\gamma}-x^{\alpha}\right|
$$

and it follows that the small perturbation $\varepsilon$ is unstable as $\beta$ increases when the difference in concentration between the two phases in equilibrium $\left|x^{\gamma}-x^{\alpha}\right|$ decreases with increasing $\beta$. The practical consequence of this observation is that integrating equation (29) from high temperature to low temperature yields a method more robust to statistical noise, because $\left|x^{\gamma}-x^{\alpha}\right|$ usually increases with decreasing temperature $T$.

We now address the second robustness issue, namely, what can we do if the algorithm wanders outside of the region of metastability of either phase? It is straightforward to detect when this happens by simply checking whether $\left|x^{\gamma}-x^{\alpha}\right|$ is not statistically significantly different from zero. One then needs to determine which of the two MC simulations (for phase $\alpha$ or $\gamma$ ) underwent a phase transition. This can be accomplished by keeping track of the concentration of each phase $\left(x_{1}^{\alpha}, \ldots, x_{n}^{\alpha}\right.$ and $\left.x_{1}^{\gamma}, \ldots, x_{n}^{\gamma}\right)$ at temperatures previously visited $\left(\beta_{1}, \ldots, \beta_{n}\right)$ and using them the verify whether the new concentration $x_{n+1}^{\alpha} \approx x_{n+1}^{\gamma}$ at $\beta_{n+1}$ is best predicted by the sequence $x_{i}^{\alpha}$ or $x_{i}^{\gamma}$, using the extrapolation described in the previous section (see figure 5). The sequence that gives the worst prediction will point to the MC simulation that underwent a phase transition.

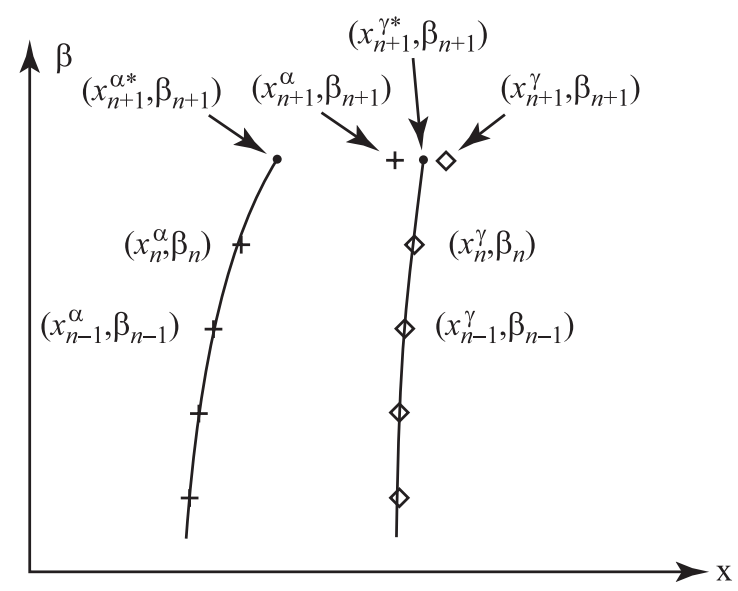

Figure 5. Criterion for detecting when the phase boundary tracing algorithm has exited the region of simultaneous metastability of the two phases in presence. The point $\left(x_{n+1}^{\alpha}, \beta_{n+1}\right)$ is better predicted by $\left(x_{n+1}^{\gamma *}, \beta_{n}\right)$ than by $\left(x_{n+1}^{\alpha *}, \beta_{n}\right)$. Hence, the MC simulation of phase $\alpha$ must have undergone a transition to phase $\gamma$. 


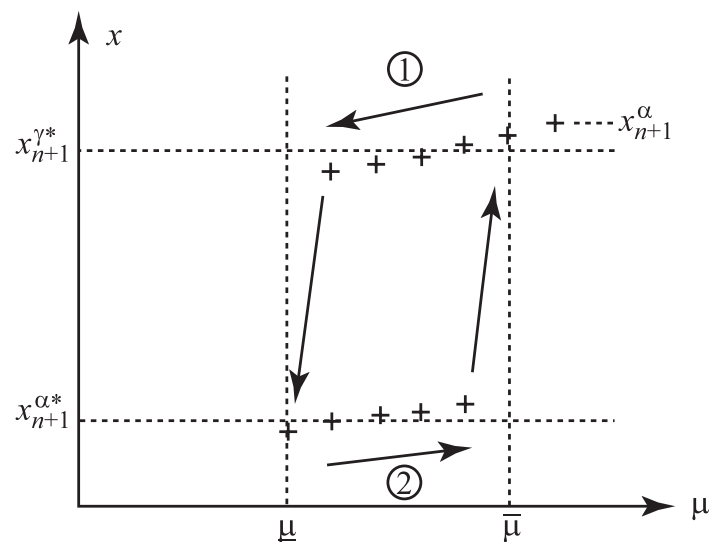

Figure 6. Algorithm to locate the centre of the region of simultaneous metastability of the two phases in presence. In step 1 , the chemical potential $\mu$ in the simulation of phase $\alpha$ is gradually decreased until concentration is once again best predicted by $x_{i}^{\alpha *}$. In step 2 , the chemical potential is increased until the simulation transform back to phase $\gamma$. The two extreme values of the chemical potential $\mu$ and $\bar{\mu}$ thus defined bracket the true value of $\mu$ at the phase transition.

Without loss of generality consider the case when $x^{\alpha}<x^{\gamma}$ and when the simulation of phase $\alpha$ transformed to phase $\gamma$. We can then 'recentre' the chemical potential to a good estimate of its true value at the phase transition as follows (refer to figure 6).

(a) Gradually decrease the chemical potential of the MC simulation of phase $\alpha$ until its concentration can again be predicted by the sequence $x_{i}^{\alpha}$ within the magnitude of the statistical noise. Let $\underline{\mu}$ denote the value of the chemical potential when this happens and save the state $S$ of the simulation cell at that point.

(b) Then, gradually increase the chemical potential of the MC simulation of phase $\alpha$ until its concentration can be predicted by the sequence $x_{i}^{\gamma}$ within the magnitude of the statistical noise. Let $\bar{\mu}$ denote the value of the chemical potential when this happens.

(c) Restore the state of the simulation cell of phase $\alpha$ to the saved state $S$ and set the chemical potential to $\mu=(\bar{\mu}+\mu) / 2$.

(d) Rerun the simulation for both phases $\alpha$ and $\gamma$ at chemical potential $\mu$ and continue the integration of equation (29) with the values of $E$ and $x$ obtained for each phase.

This approach results in a very robust algorithm that can run without user intervention to map out the whole two-phase equilibrium. Thanks to this safety mechanism, we found that it is feasible to integrate equation (29) towards increasing temperature, despite the potential for instability with respect to noise. This proves useful since it is very easy to find a starting point for our algorithm at $T=0$. The boundary tracing algorithm may have to recentre itself a few times on its way to the end of the two-phase equilibrium. But once the location of the phase boundary at high temperature is known, a smooth phase boundary can then be calculated, starting from the highest temperature and following the phase boundary in the direction of decreasing temperature.

The end of the two-phase region can be detected by monitoring the occurrence of either one the following events (see figure 7):

(a) the concentrations $x^{\alpha}$ and $x^{\gamma}$ are statistically significantly different, but either $x^{\alpha}$ or $x^{\gamma}$ is not predicted by the corresponding sequence $x_{i}^{\alpha}$ or $x_{i}^{\gamma}$ within statistical uncertainty; 

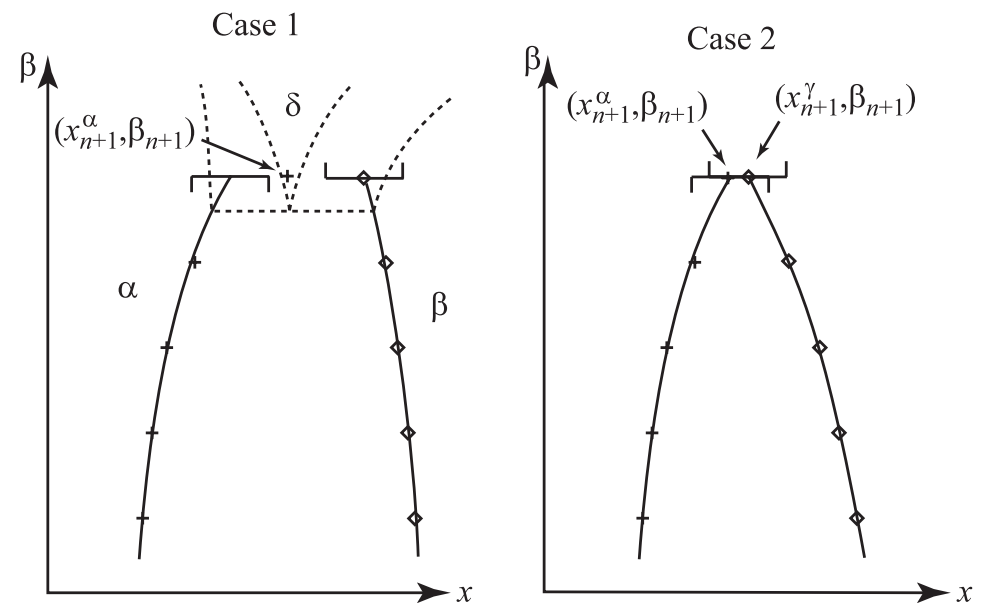

Figure 7. Criterion for detecting the end of a two-phase equilibrium. In case 1, a new phase $\delta$ has appeared, as indicated by the fact that $x_{n+1}^{\alpha}$ lies outside of the error bar of the prediction of concentration for either phase $\alpha$ or $\gamma$. In case 2, a congruent or critical point has been reached since the concentrations of the two phases are no longer statistically significantly different.

(b) the concentrations $x^{\alpha}$ and $x^{\gamma}$ are not statistically significantly different, but the sequences $x_{i}^{\alpha}$ and $x_{i}^{\gamma}$ both predict the same concentration, within statistical uncertainty.

Case 1 indicates that a third phase $\delta$ has become stable (i.e. a eutectic or peritectic point), while case 2 indicates that the phase boundaries have met at a common composition (e.g. at a congruent point or a critical point). In the first case, the algorithm will actually continue slightly beyond the true eutectic or peritectic point because of the presence of hysteresis at a first-order phase transition. However, the true location of the three-phase equilibrium can be determined from the intersection of the $\alpha-\delta$ and $\delta-\gamma$ phase boundaries, once they have been determined.

\section{Applications}

This section presents a number of examples of automated calculations of thermodynamic properties using the previously introduced algorithms. In each example, the cluster expansion describing the energetics of the alloy system was obtained from a fit to first-principles structural energy calculations using the MAPS [32] code, which automates the process. A description of the algorithms enabling the automatic construction of such a cluster expansion, as well as a description of the interaction parameters defining each cluster expansion used below, can be found in [33].

Our first example is the determination of the complete potential surface $\phi(T, \mu)$ of the solid-state phases of the $\mathrm{MgO}-\mathrm{CaO}$ pseudo-binary alloy system (see figure 8 ). As the $\mathrm{MgO}-\mathrm{CaO}$ system is phase separating (as shown in figure 12), the thermodynamic potential surface exhibits a characteristic butterfly shape. Below the critical temperature, the surface intersects with itself at a nonzero angle, indicating the presence of a miscibility gap. Since the MC simulation is able to sample metastable phases, for some values of the chemical potential, one can determine $\phi(T, \mu)$ for both phases in the region of metastability, which explains the double-valued nature of the potential surface at low temperatures. Above the critical temperature, $\phi(T, \mu)$ becomes single-valued and varies smoothly as $\mu$ varies. 


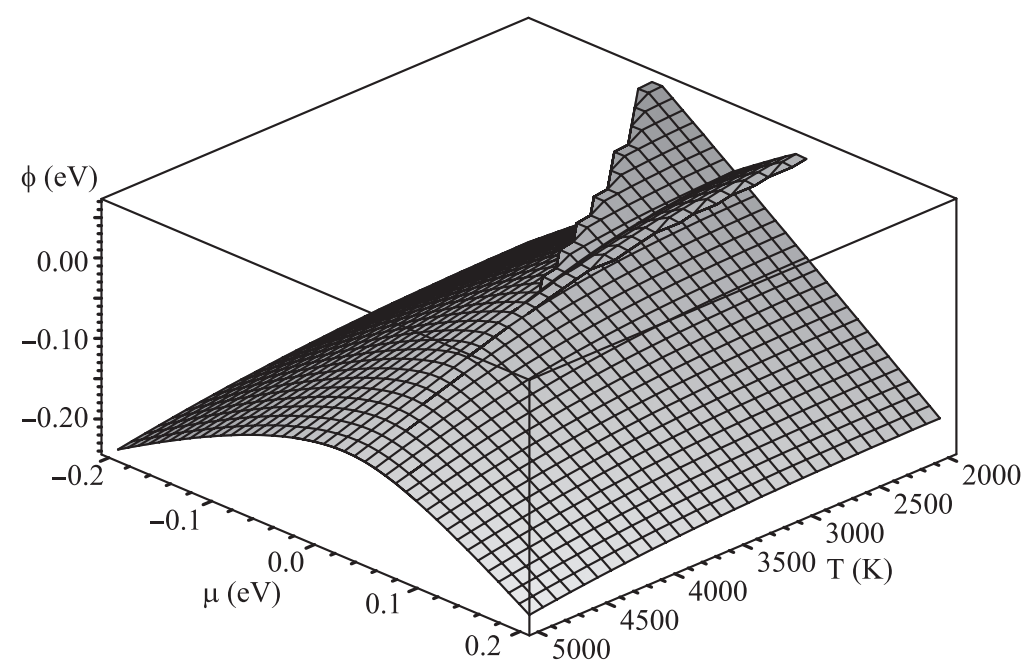

Figure 8. Potential surface for the $\mathrm{MgO}-\mathrm{CaO}$ pseudo-binary alloy system.

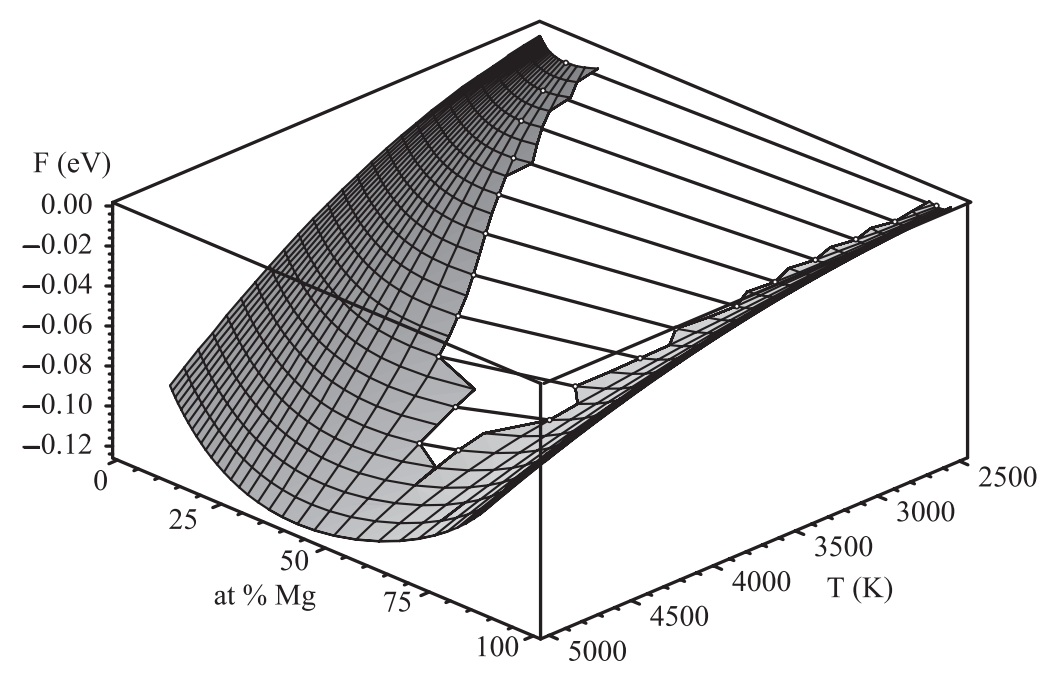

Figure 9. Gibbs free energy for the $\mathrm{MgO}-\mathrm{CaO}$ pseudo-binary alloy system. Common tangent constructions are shown at selected temperatures.

(Note that, in reality, the alloy melts before reaching that point at atmospheric pressure.) Figure 9 shows the Gibbs ${ }^{6}$ free energy surface $G(x, T) \equiv \phi(T, \mu(T, x))+\mu(T, x) x$ for the same system and, in this more familiar representation, the presence of miscibility gap at low temperature is clearly recognizable. The calculated Gibbs free energy surface can be directly used to fit standard solution models, such as an ideal solution model, plus a polynomial in temperature and concentration to account for non-ideality. The order of the polynomial needed to represent the MC output can, for instance, be determined through cross-validation, as defined in section 3.2. The resulting very compact representation of the thermodynamics of the alloy

6 The difference between the Gibbs and Helmholtz free energies can be neglected at atmospheric pressure in solid-state systems because the product of pressure $P$ with changes in specific volume $(\Delta V)$ are small. 


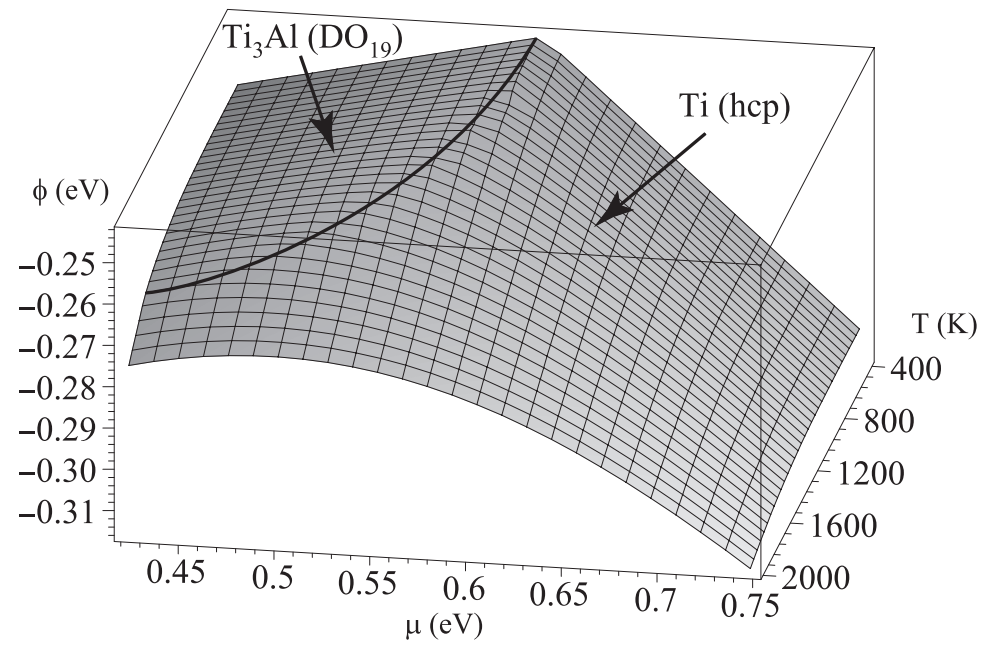

Figure 10. Potential surface for the Ti-Al alloy system. The thick curve indicates the location of a kink in the surface, associated with a first-order transition.

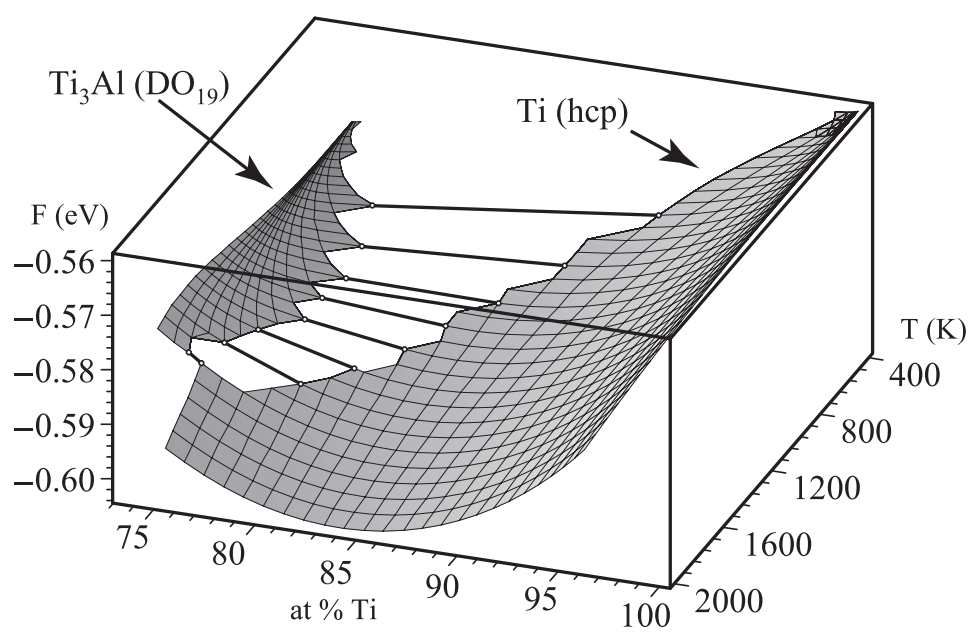

Figure 11. Gibbs free energy for the Ti-Al alloy system. Common tangent constructions are shown at selected temperatures.

system can be used in CALPHAD-type calculations to supplement existing experimental data or to fill in data not yet determined experimentally.

Figure 10 shows the result of a similar calculation in the case of a ordering alloy system, the Ti-Al system. For clarity, the metastable portions of the $\phi(T, \mu)$ surface are omitted so that the potential is single-valued everywhere. The order-disorder transition is clearly visible from the kink in the surface, which is highlighted by a thick curve. The corresponding Gibbs free energy representation of the same data is shown in figure 11.

Figure 12 illustrates the usefulness of the boundary tracing algorithm described in section 3.3. The examples shown even include a case (Ti-Al) where a equilibrium between two phases differing by their lattice type: the $\mathrm{L} 1_{0}$ is fcc-based while the $\mathrm{DO}_{19}$ and the Ti solid solution are hcp-based). These examples show that one can obtain phase diagrams from first principles without first obtaining the complete $\phi(T, \mu)$ surface. 

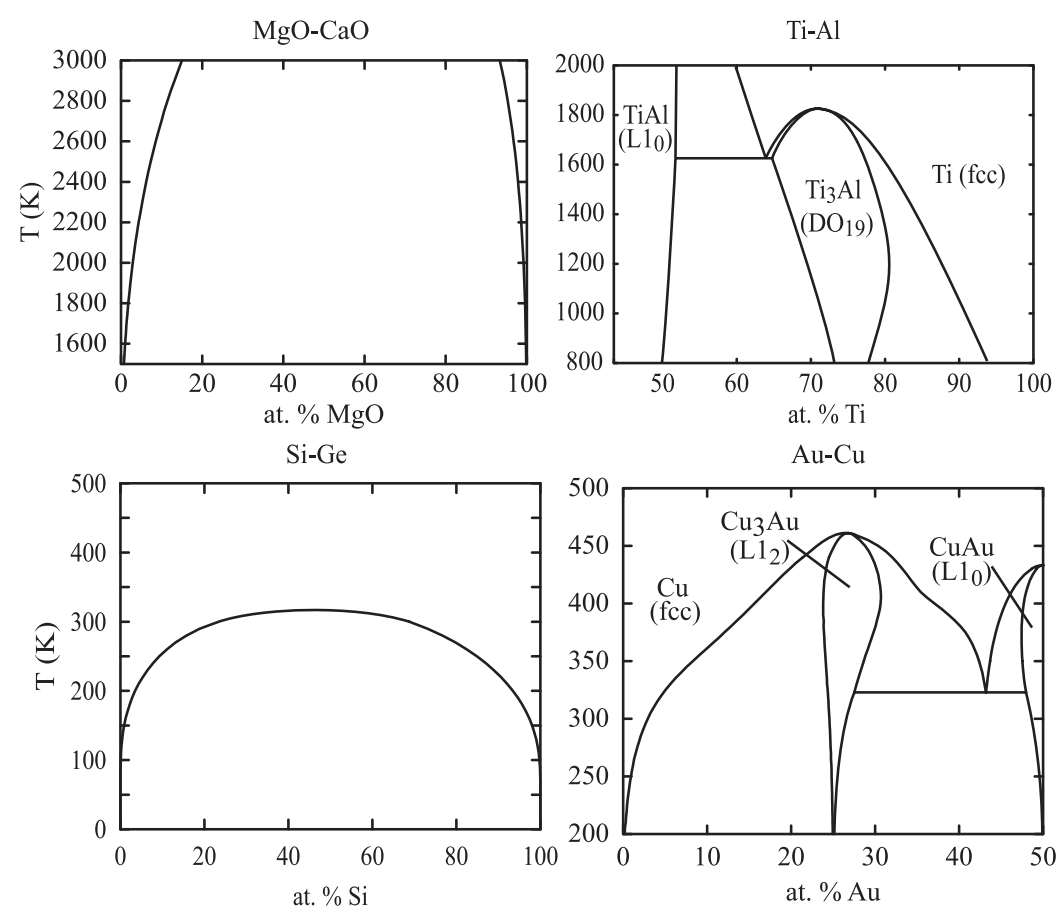

Figure 12. Sample phase diagrams calculated with the automatic boundary tracing algorithm.

It is important to note that, in these examples, the MC code requires very little intervention or input from the user. Thanks to the algorithms introduced in section 3.1, the code is able to autonomously determine, at each value of the control variables $(T, \mu)$, when equilibrium has been reached and how long the simulation needs to be run in order to reach the target precision. The code is also able to determine when the region of stability of one phase has been exited, thanks to the algorithms described in section 3.2, so that the user does not need to specify when to stop the thermodynamic integration. The only input parameters the user needs to specify are:

(a) which phase to scan (the code can determine the value of the chemical potential $\mu$ stabilizing the desired two-phase equilibrium at $0 \mathrm{~K}$ );

(b) the target precision (here, 0.1 at.\% precision on the concentration variable is used as a criterion);

(c) the size of the simulation cell (in this study, the simulation cell is always chosen such that it contains a sphere of a diameter equal to at least 10 times the nearest-neighbour interatomic spacing).

\section{Conclusion}

The algorithms we have introduced enable researchers to focus on high-level concepts when employing MC simulations. All the tasks that make traditional MC simulations so tedious have been formalized into algorithms that can autonomously initialize and control the appropriate MC simulation. The user only needs to specify high-level goals, such as the target precision or which phase to focus on. This automation of MC simulations, along with the earlier work that 
enabled the automation of cluster expansion construction [33], signifies that it is now possible for large community of researchers to employ first-principles calculations to augment and complete existing experimental thermodynamic databases without facing the steep learning curve that has traditionally been associated with first-principles thermodynamical calculations.

\section{Acknowledgment}

This work was supported by the NSF under program DMR-0080766.

\section{References}

[1] Newman M E J and Barkema G T 1999 Monte Carlo Methods in Statistical Physics (Oxford: Clarendon)

[2] Binder K and Heermann D W 1988 Monte Carlo Simulation in Statistical Physics (New York: Springer)

[3] Dünweg B and Landau D P 1993 Phys. Rev. B 4814182

[4] Laradji M, Landau D P and Dünweg B 1995 Phys. Rev. B 514894

[5] Sanchez J M, Ducastelle F and Gratias D 1984 Physica A 128334

[6] de Fontaine D 1994 Solid State Phys. 4733

[7] Zunger A 1994 NATO ASI on Statics and Dynamics of Alloy Phase Transformation vol 319, ed P E Turchi and A Gonis (New York: Plenum) p 361

[8] Jones R O and Gunnarsson O 1989 Rev. Mod. Phys. 61689

[9] de Fontaine D 1979 Solid State Phys. 3474

[10] Laks D B, Ferreira L G, Froyen S and Zunger A 1992 Phys. Rev. B 4612587

[11] Ducastelle F 1991 Order and Phase Stability in Alloys (New York: Elsevier)

[12] Ceder G 1993 Comput. Mater. Sci. 1144

[13] Wolverton C and Zunger A 1995 Phys. Rev. Lett. 753162

[14] Asta M and Foiles S M 1996 Phys. Rev. B 532389

[15] van de Walle A and Ceder G 2002 Rev. Mod. Phys. 7411

[16] Ozolinsš V and Asta M 2001 Phys. Rev. B 86448

[17] Ozoliņš V, Wolverton C and Zunger A 1998 Phys. Rev. B 58 R5897

[18] Wolverton C and Ozolinsš V 2001 Phys. Rev. Lett. 865518

[19] Wolverton C and Zunger A 1995 Phys. Rev. B 528813

[20] Kikuchi R 1951 Phys. Rev. 81988

[21] Lu Z W, Laks D B, Wei S-H and Zunger A 1994 Phys. Rev. B 506642

[22] Zunger A 1997 MRS Bull. 2220

[23] Wolverton C, Ozolinš̌ V and Zunger A 2000 J. Phys.: Condens. Matter 122749

[24] Ceder G, van der Ven A, Marianetti C and Morgan D 2000 Modelling Simul. Mater. Sci. Eng. 8311

[25] Asta M, Ozolins V and Woodward C 2001 J. Minerals Metals Mater. Soc. 5316

[26] van de Walle A 2001 Easy Monte Carlo Code (Emc2), http://cms.northwestern.edu/atat/

[27] Kohan A F, Tepesch P D, Ceder G and Wolverton C 1998 Comput. Mater. Sci. 9389

[28] Woodward C, Asta M, Kresse G and Hafner J 2001 Phys. Rev. B 63094103

[29] Stone M and Roy J 1974 Stat. Soc. Met. B 36111

[30] Li K-C 1987 Ann. Stat. 15956

[31] Goldberger A S 1991 A Course in Econometrics (Cambridge, MA: Harvard University Press)

[32] van de Walle A 2001 MIT Ab-initio Phase Stability (MAPS) code, http://www.mit.edu/avdw/maps/

[33] van de Walle A and Ceder G 2001 Automating first-principles phase diagram calculations J. Phase Equilibria at press 